\title{
Influence du type de station et du traitement sylvicole sur la qualité du bois de hêtre dans le Nord-Est Essai d'explication de certaines caractéristiques physiques par la structure du plan ligneux
}

\author{
J.-P. PREISS \\ I.N.R.A. Station de Recherches sur la Qualité des Bois \\ Centre de Recherches forestières de Nancy/Champenoux - F 54280 Seichamps
}

Mémoire de $3^{\circ}$ année de $1^{\prime}$ Ecole Nationale des Ingénieurs des Travaux des Eaux et des Foiêts Septembre 1981

Cette étude fait suite à de nombreux travaux déjà réalisés dans le Nord-Est de la France par la Station de Recherches sur la Qualité des Bois pour essayer de définir les stations forestières et le type de sylviculture les mieux à même de produire du bois de hêtre de bonne qualité technologique, c'est-à-dire du bois à faible niveau de contraintes de croissance, à densité et à rétractibilité peu élevées.

Dans un premier temps, est abordé l'aspect phyto-écologique qui doit permettre de mieux caractériser les stations à hêtre de qualité. Cinquante relevés floristiques ont été réalisés dans différentes placettes de taillis-sous-futaie couvrant toute la variabilité édaphique des hêtraies de plaine du Nord-Est. Un tableau diagonalisé a été construit, qui permet de distinguer 10 groupes écologiques et 4 grands types de stations forestières allant des hêtraies-chênaies xérophiles sur sol carbonaté superficiel aux hêtraies-chênaies acidophiles. Par ailleurs, l'étude d'un échantillonnage de relevés répartis également entre futaie et taillis-sous-futaie a montré que le type de traitement semblait exercer sur la flore un rôle secondaire par rapport au type de sol.

L'étude de la variabilité de la qualité du bois en fonction du type de station et du type de sylviculture est alors entreprise à partir d'essais technologiques permettant d'évaluer les contraintes de croissance, et de déterminer les retraits, ainsi que l'infradensité du bois, exclusivement sur des carottes de sondage, en utilisant donc une méthodologie non destructive.

Dans une première étude sont traités séparément un échantillonnage de 38 placettes de futaie et un échantillonnage de 49 placettes de taillis-sous-futaie. Les principaux résultats sont les suivants :

- effet du type de station : en taillis-sous-futaie, l'infradensité et la rétractibilité augmentent parallèlement au gradient de fertilité stationnel, mais aucun effet comparable n'apparaît en futaie ;

- variabilité : les variances entre moyennes de placettes sont plus fortes en futaie qu'en taillis-sous-futaic. Ceci résulte sans doute des fortes différences d'âge 
existant entre certaines placettes de futaie. En revanche, les variances intraplacettes sont du même ordre de grandeur pour les deux traitements;

- liaisons entre caractères : pour les deux traitements, infradensité, largeur de cerne et retrait volumétrique élevés vont de pair. Au niveau interplacettes, l'infradensité augmente parallèlement à la largeur de cerne; en revanche, au niveau intraplacette, en futaie, l'infradensité est indépendante de la largeur de cerne.

Une autre comparaison futaie - taillis-sous-futaie repose sur un échantillonnage de 40 placettes réparties à effectifs égaux entre les deux types de traitements et quatre types de stations forestières. L'étude de cet échantillonnage a permis de mettre en évidence les influences respectives du type de traitement sylvicole et du type de station sur certaines caractéristiques du bois : les contraintes de croissance, le retrait axial et le retrait radial sont essentiellement influencés par le type de sylviculture; leurs valeurs sont plus faibles en taillis-sous-futaie; le retrait tangentiel et la largeur de cerne sont surtout influencés par le type de station; leurs valeurs augmentent avec le gradient de fertilité stationnel; l'infradensité est influencée de manière significative par les deux facteurs à la fois. L'interaction type de station - traitement n'est jamais significative.

Dans la dernière partie du travail, un essai d'explication de certaines caractéristiques technologiques par la structure du plan ligneux est tenté à partir d'un échantillonnage de 110 carottes présentant des valeurs extrêmement fortes ou extrêmement faibles pour les caractéristiques suivantes : largeur de cerne, infradensité, retraits tangentiel, radial, axial ; en outre, 10 carottes à retrait axial quasiment nul ont été sélectionnées. Des mesures densitométriques complètent les données d'infradensité, et une quantification de la structure anatomique (pourcentage de vaisseaux, de fibres normales et de parenchyme, de fibres de bois de tension, de rayons ligneux en coupe transversale et en coupe tangentielle) est obtenue sur un analyseur d'images.

Les résultats concordent dans l'ensemble avec ceux de la littérature; ils indiquent que les échantillons à forte largeur moyenne de cerne ont un faible pourcentage de vaisseaux, un fort pourcentage de fibres, une infradensité et une rétractabilité élevées; le bois de tension semble apparaître essentiellement au niveau des cernes larges; sa présence s'accompagne de fortes valeurs positives du retrait axial. Aucune liaison entre le pourcentage de rayons ligneux et les caractéristiques physiques du bois ne peut être mise en évidence. 